\title{
O ENVELHECIMENTO COMO FATOR DETERMINANTE NAS MODIFICAÇÕES DA ESTRUTURA RENAL
}

\section{THE AGEING DETERMINATED FACTOR ON THE KIDNEY ARCHITECTURE CHANGES}

\author{
Juliana Regina Dias Lemos ${ }^{1}$; Javier Emílio Lazo Chica²; Gustavo Lemos Campos \\ Sousa $^{3}$; Marlene Antônia dos Reis ${ }^{2}$; Vicente de Paula Antunes Teixeira ${ }^{2}$ \\ 1 Autor para contato: Universidade Estadual de Ponta Grossa, Departamento de \\ Enfermagem e Saúde Púbica, Ponta Grossa-PR, Brasil; (42) 3220-3735; \\ e-mail: lemos.jrd@terra.com.br \\ 2 Universidade Federal do Triângulo Mineiro - UFTM, Uberaba - MG \\ 3 Aluno do Programa de Pós-graduação em Patologia da UFTM
}

Recebido para publicação em 23/05/2008

Aceito para publicação em 06/11/2008

\section{RESUMO}

O envelhecimento envolve alterações histológicas e fisiológicas, em vários órgãos, em muitos indivíduos, com idades entre 30 e 85 anos, podendo ocorrer uma redução de 20-25\% do conteúdo renal, além de mudanças estruturais importantes, como redução do peso renal de 10 a 43\%, até a idade de 80 anos. O objetivo do estudo foi avaliar alterações da estrutura renal durante o envelhecimento, sendo selecionados 38 pacientes, ausentes, de nefropatias primárias e secundárias, no diagnóstico pós mortem, na faixa etária de 20 a 79 anos, que vieram a óbito no Hospital Universitário da Universidade Federal do Triângulo Mineiro. Durante o exame de autópsia, ao mesmo tempo em que a antropometria do indivíduo era realizada, foram retirados os rins direito e esquerdo, para pesar e para fazer demais análises histológicas, sendo constatada correlação negativa e significativa entre o peso renal total e a idade dos pacientes, bem como entre o peso dos rins direito e esquerdo e a idade. Além disso notou-se correlação positiva e significativa entre o peso renal total e o peso corporal, e entre o peso renal total e o IMC, sendo que, com relação ao colágeno, a faixa etária de pacientes idosos apresentou maior porcentagem de fibrose, tanto no rim direito quanto no rim esquerdo, quando comparados aos pacientes adultos jovens e adultos. O estudo propicia entender as possíveis alterações macroscópicas que os rins podem sofrer no envelhecimento fisiológico, uma vez que alterações de caráter microscópico já são observadas nos quatro compartimentos renais, principalmente no interstício.

Palavras chave: Rim. Envelhecimento. Peso renal. Fibrose intersticial.

\begin{abstract}
Aging involves biological and physiological alterations in various organs in a great number of individuals aged between 30 and 85 . A reduction of $20-25 \%$ of renal


contents, besides important structural changes, such as a reduction of between $10 \%$ to $43 \%$ of kidney weight up to the age of 80 can occur. The aim of this study was to evaluate alterations in the renal structure during the aging process. 38 patients aged between 20 and 79, with no primary or secondary nephropathies in post-mortem diagnosis, were selected, all of them having died in the University Hospital of the "Universidade Federal do Triângulo Mineiro". During autopsy, at the same time as the anthropometry of the individual was performed, the kidneys were removed to be weighed and biologically analyzed. The results showed significant negative correlation between the total renal weight and the ages of the patients, as well as between the weight of the right and left kidneys and the patients' age. There was also a positive and significant correlation between the total renal weight and the corporal weight, and between the total renal weight and the CMI. As to collagen, elderly patients presented a higher percentage of fibrosis in the right as well as in the left kidney, when compared to those of young-adult and adult patients. Thus, this study evinced the possibility of macroscopic alterations kidneys can undergo during physiological aging, for microscopic alterations can already be observed in the four renal compartments, mainly in the interstice.

Keywords: Kidney. Aging. Renal weight. Interstitial fibrosis.

\section{Introdução}

O século XX foi marcado por um fenômeno denominado explosão demográfica, já que evidenciou um crescimento expressivo da população mundial, que chegou a atingir seis milhões de pessoas no ano 2000. Para mensurar de maneira mais precisa, dados demográficos revelam que a população mundial aumentou em três milhões pessoas entre os anos de 1960 e 2000 (CORBINOS, 2001).

Os países do chamado "Terceiro Mundo” vêm apresentando, nas duas últimas décadas, um progressivo declínio das suas taxas de mortalidade e, mais recentemente, nas taxas de fecundidade. Esses dois fatores associados promovem a base demográfica para um envelhecimento real dessas populações. Essa transição demográfica traduz-se na elevação da expectativa de vida média da população e no aumento em termos absolutos e proporcionais do número de pessoas atingindo idade avançada (AMARAL, COELI; COSTA et al., 2004).

Projeções recém-publicadas pela Organização Mundial de Saúde (OMS) estimam que, entre 1990 e 2025, a população idosa aumentará de sete a oito vezes em países como a Colômbia. As mesmas projeções indicam que, entre os dez países com as maiores populações idosas em 2025, cinco serão países em desenvolvimento, incluindo o Brasil com um número estimado de 27 milhões de pessoas com sessenta anos ou mais (AMARAL, COELI; COSTA et al., 2004).

O envelhecimento é uma conseqüência inevitável da vida que envolve alterações histológicas e fisiológicas em vários órgãos, e há aproximadamente quatro décadas foram documentadas alterações relacionadas aos rins que envolviam esclerose generalizada com conseqüente comprometimento da função renal (JASSAL; OREOPOULOS, 1998).

Em relação às mudanças estruturais, descreveu-se que o tamanho e o peso renais apresentam redução de 10 a $43 \%$ até à idade de 80 anos. Microscopicamente, o número de glomérulos e de túbulos proximais diminui se comparado com a massa renal total. Essa diminuição é mais expressiva na região cortical. As mudanças glomerulares aparecem gradualmente com alterações na membrana basal glomerular (MBG), seguidas por depósitos hialinos e eventual esclerose deste compartimento. Já no compartimento intersticial ainda podem ser observados o aumento da síntese e/ou a redução da degradação de fibras colágenas do tipo I, II e II, integrantes da matriz gelatinosa, também composta por proteoglicanos, glicoproteínas (fibronectina e laminina) e líquido intersticial, o que favorecerá 
o acúmulo de colágeno e conseqüente processo de fibrose intersticial (LEMLEY; KRIZ, 1991).

O envelhecimento também parece estar associado com o aumento de células mesangiais do capilar glomerular, acompanhadas por uma maior expressão de tecido mesangial, que aumenta de $8 \%$ do volume total glomerular, aos 45 anos, para aproximadamente $12 \%$, aos 70 anos (JASSAL; OREOPOULOS, 1998).

Rins de indivíduos idosos normais, com declínio na taxa de filtração glomerular, apresentam também uma redução em outros mecanismos homeostáticos renais, que parecem estar bastante ligados a um grande número de nefrites intersticiais crônicas (BECK, 2000).

\section{Materiais e métodos}

O projeto foi aprovado pelo Comitê de Ética em Pesquisa (CEP) da Universidade Federal do Triângulo Mineiro com o protocolo de n. 468. Foram estudados retrospectivamente os laudos de autopsia feitas no HE-UFTM, entre janeiro de 2000 e julho de 2004. Foram incluídos todos os laudos de indivíduos cujo exame da autópsia não demonstrou alterações renais compatíveis com algum quadro de nefropatia primária, caracterizadas por alterações renais causadas por lesões primárias encontradas em qualquer compartimento do parênquima renal, como as glomerulopatias, nefrites túbulo intersticiais e vasculopatias, ou secundária, definidas por alterações renais causadas por doenças sistêmicas com capacidade de lesar áreas do parênquima secundariamente, como diabetes mellitus, lúpus eritematoso sistêmico, hepatites e doenças inflamatórias crônicas. Foram excluídos todos os laudos de indivíduos com idade inferior a 20 anos e alterações renais do tipo congestão renal e enfartos renais. Realizamos a separação das peças anatômicas renais que correspondiam aos casos amostrais e efetuamos as pesagens destes antes dos procedimentos de preparação histológica. As informações referentes ao biótipo humano de cada indivíduo foram retiradas dos laudos de autópsia, uma vez que estes dados já são coletados precisa- mente durante o exame pós mortem. O material histológico, rins direito e esquerdo, retirados no momento da autópsia, foram seccionados em cortes com espessura de $3 \mu \mathrm{m}$ e posteriormente submetidos à coloração pelo Picro-sírius. Em seguida, para análise morfométrica, os cortes correspondentes aos terços superior, médio e inferior de ambos os rins foram examinados sob luz polarizada, com objetiva de 10x e aumento final de 600 vezes, utilizando um sistema analisador de imagens automático (KS 300 Carl Zeiss ${ }^{\circledR}$ ). As variáveis quantitativas foram testadas para verificar se tinham distribuição normal através do teste de Kolmogorov-Smirnov. Em casos de distribuição normal e variâncias semelhantes, foram utilizados testes paramétricos: o teste " $t$ " de Student, na comparação entre dois grupos, e o teste ANOVA (F), para comparação entre três ou mais grupos, seguido do teste de Tukey ou Bonferroni, quando necessário. Neste tipo de distribuição, os resultados foram expressos em média \pm desvio padrão (X $\pm \mathrm{DP})$. Em casos de distribuição não normal, os resultados foram expressos em mediana e valores mínimos e máximos (Med - min-max). Nesta condição ou quando a distribuição for normal, porém com variâncias não semelhantes entre os grupos, os testes utilizados foram os não paramétricos: o teste de Mann-Witney ( $\mathrm{T}$ ) na comparação entre dois grupos, e o teste de Kruskal-Wallis (H), na comparação entre três ou mais grupos, seguido pelo teste de Dunn, quando necessário. A correlação entre duas variáveis, com distribuição normal e não normal foram analisadas pelos testes de Pearson (r) e Spearman (rS), respectivamente. Foram consideradas estatisticamente significativas as diferenças em que a probabilidade (p) foi menor que $5 \%(p<0,05)$.

\section{Resultados}

\section{Análise descritiva geral}

Dos 38 laudos de autópsia de indivíduos sem nefropatias, observou-se predomínio do gênero masculino, em 24 (63\%) dos casos, e da cor branca, em 22 (58\%) dos casos. A média das idades foi de $42,7 \pm 16,5$ anos, a idade masculina correspondeu a 
Tabela 1 - Comparação entre a idade, a altura e o Índice de Massa Corporal (IMC) com o gênero de 38 pacientes autopsiados no HE-UFTM, Uberaba (MG), no período de janeiro de 2000 a julho de 2004.

\begin{tabular}{|c|c|c|c|c|}
\hline & Idade & IMC & Altura & n (\%) \\
\hline \multicolumn{5}{|c|}{$X \pm D P$} \\
\hline Masculino & $42,7 \pm 16,7$ & $21,5 \pm 6,3$ & $1,69 \pm 0,07$ & $24(63)$ \\
\hline Feminino & $42,6 \pm 16,8$ & $21,2 \pm 2,7$ & $1,55 \pm 0,08$ & $14(37)$ \\
\hline Total & & & & $38(100)$ \\
\hline
\end{tabular}

Idade: $\mathrm{t}=0,0195 ; \mathrm{p}=0,985 /$ IMC: $\mathrm{t}=0,281 ; \mathrm{p}=0,781 /$ Altura: $\mathrm{t}=5,377 ; \mathrm{p}=<0,001$

$\mathrm{n}=\mathrm{n}^{\mathrm{o}}$. de pacientes.

Tabela 2 - Comparação entre peso corporal, peso do rim total e dos rins direito e esquerdo com o gênero de 38 pacientes autopsiados no HE-UFTM, Uberaba (MG), no período de janeiro de 2000 a julho de 2004.

\begin{tabular}{lccccc}
\hline & Peso corporal & Peso renal total & Rim direito & Rim esquerdo & n (\%) \\
& Med(mín-máx) & $\boldsymbol{X} \pm \boldsymbol{D P}$ & $\boldsymbol{X} \pm \boldsymbol{D P}$ & $\boldsymbol{X} \pm \boldsymbol{D P}$ & \\
\hline Masculino & $57(45-77)$ & $370,2 \pm 134,0$ & $181,6 \pm 67,5$ & $188,6 \pm 68,3$ & $24(63)$ \\
Feminino & $52,5(44-56,5)$ & $301,2 \pm 81,3$ & $150,1 \pm 43,0$ & $151,1 \pm 40,6$ & $14(37)$ \\
\hline Total & & & & & $38(100)$ \\
\hline
\end{tabular}

Peso corporal: $\mathrm{t}=226,5 ; \mathrm{p}=0,164$ / Peso renal total: $\mathrm{t}=1,743 ; \mathrm{p}=0,090 /$ Rim direito: $\mathrm{t}=1,565 ; \mathrm{p}=0,126$ /

Rim esquerdo: $t=1,862 ; p=0,071 . n=n^{0}$. de pacientes.

$42,7 \pm 16,7$ anos e a feminina, a $42,6 \pm 16,8$ anos. A altura apresentou média de $1,64 \pm 0,09$ metros entre os pacientes. $\mathrm{O}$ gênero masculino, com média de altura de 1,69 $\pm 0,07$ metros, e o gênero feminino, com $1,55 \pm 0,08$ metros, sendo estatisticamente significativa esta diferença. A média do IMC foi de $21,4 \pm 5,2 \mathrm{~kg} / \mathrm{m}^{2}$, sendo que os homens apresentaram média de $21,5 \pm 6,3 \mathrm{~kg} / \mathrm{m}^{2}$ e as mulheres, de $21,2 \pm 2,7$ $\mathrm{kg} / \mathrm{m}^{2}$, não apresentando diferença significativa entre os gêneros. O valor do IMC $<18,57 \mathrm{kKg} / \mathrm{m}^{2}$ foi encontrado em 13 casos (34\%), sendo que $10(77 \%)$ indivíduos eram do gênero masculino e 3 (23\%), do gênero feminino (Tabela 1).

\section{Análise macroscópica}

A mediana do peso corporal dos pacientes correspondeu a $57 \mathrm{~kg}$ (45-77), no gênero masculino, e a 52,5kg (44-56,5), no gênero feminino. (Tabela 2).

A média do peso renal total foi de $344,8 \pm 120,9$, sendo o peso renal total maior no gênero masculino, 370,2士134,0, em relação ao gênero feminino, 301,2 $\pm 81,3$. O peso renal direito apresentou média de $170 \pm 61 \mathrm{~g}$. No gênero masculino correspondeu a

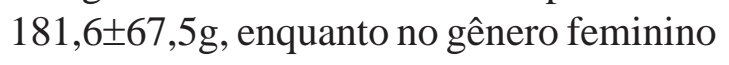

apontou para 150,1 $\pm 43,0 \mathrm{~g}$. A média do peso renal esquerdo foi maior que a do direito, $174,8 \pm 61,8 \mathrm{~g}$, e o gênero masculino também apresenta maior média de peso renal, 188,6 6 68,3g, em relação à média do

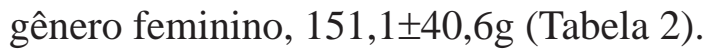

Em nossos dados houve uma correlação negativa e significativa entre e a idade e o peso renal total (Gráfico 2). Esta relação foi semelhante quando analisamos os pesos do rim direito e do rim esquerdo separadamente (Gráficos 3 e 4). 


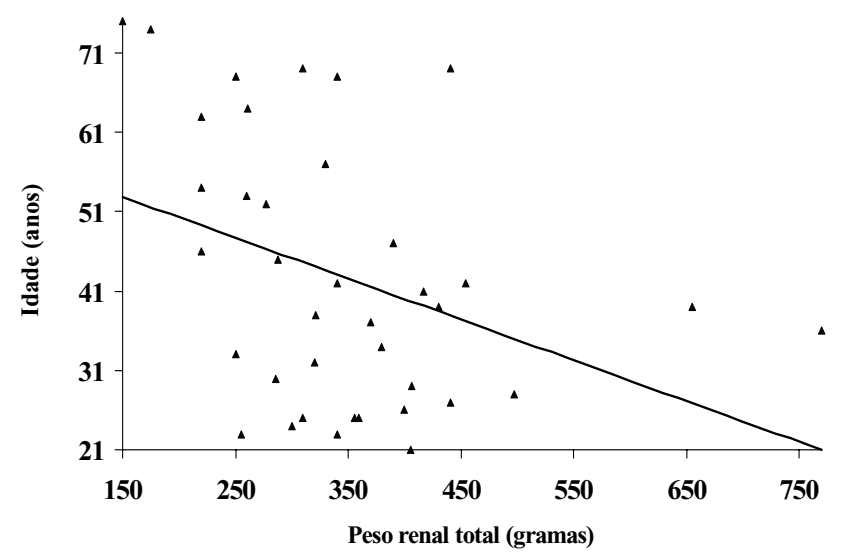

Coeficiente de correlação de Pearson $=\mathbf{- 0 , 3 6 1 ; p = 0 , 0 2 6 0}$

Granco 2 - Correlação entre a idade e o peso renal total em pacientes autopsiados sem nefropatias no HEUFTM, Uberaba (MG), no período de janeiro de 2000 a julho de 2004.

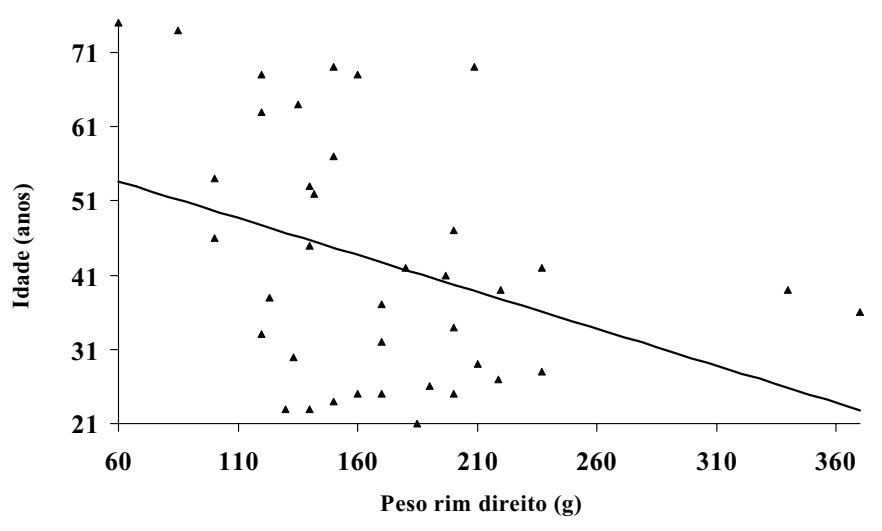

Coeficiente de correlação de Pearson $=-\mathbf{0 , 3 6 5} ; \mathbf{p}=\mathbf{0 , 0 2 3 7}$

Grático 3 - Correlação entre a idade e o peso do rim direito em pacientes autopsiados sem nefropatias no HEUFTM, Uberaba (MG), no período de janeiro de 2000 a julho de 2004.

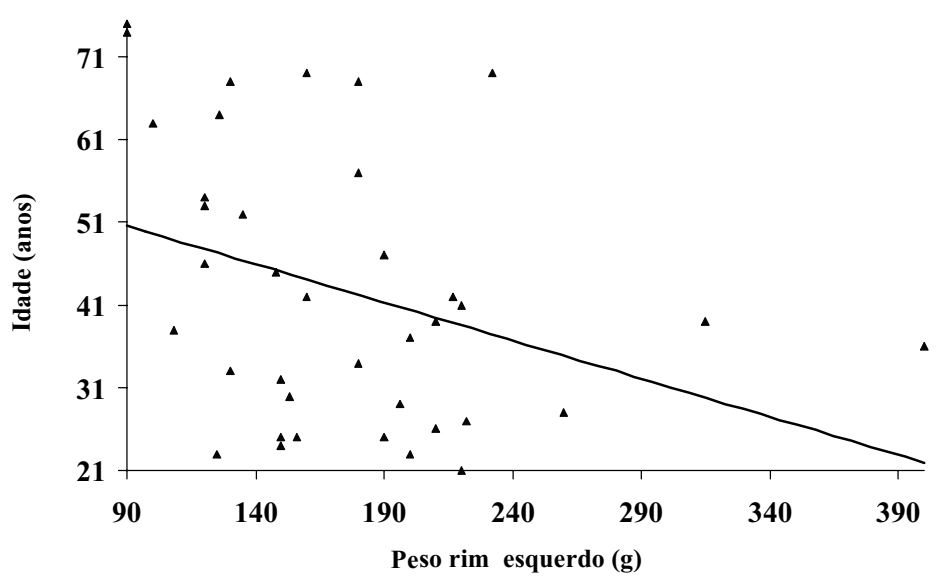

Coeficiente de correlação de Pearson $=\mathbf{- 0 , 3 4 4} ; \mathrm{p}=\mathbf{0 , 0 3 4 3}$

Gráfico 4 - Correlação entre a idade e o peso do rim esquerdo em pacientes autopsiados sem nefropatias no HE-UFTM, Uberaba (MG), no período de janeiro de 2000 a julho de 2004. 
O IMC apresentou correlação positiva e significativa com o peso renal (Gráfico 5).

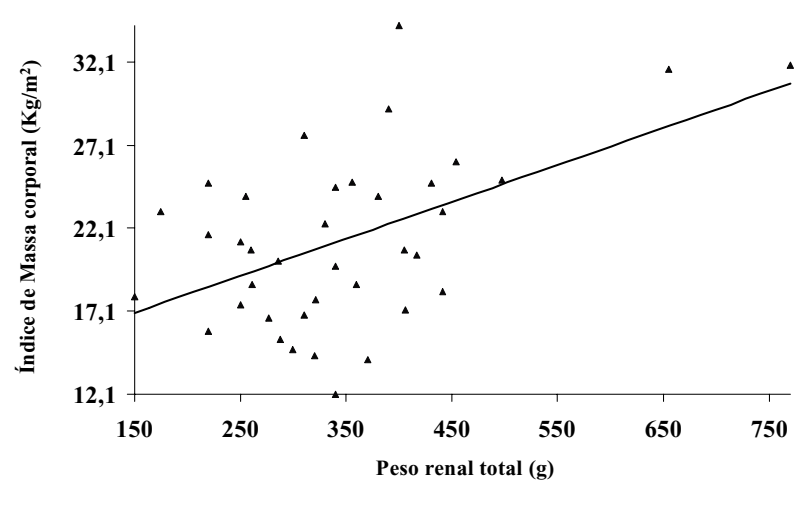

Coeficiente de correlação de Pearson = 0,516; $p=0,000901$

Gráfico 5 - Correlação entre o Indice de Massa Corporal (IMC) e o peso renal total em pacientes autopsiados sem nefropatias no HE-UFTM, Uberaba (MG), no período de janeiro de 2000 a julho de 2004.

\section{Análise microscópica}

Os resultados encontrados com relação ao colágeno mostraram que, em ambos os rins, a porcentagem de fibrose foi de 23,5 e 24,5\%, respectivamente, no rim direito e no rim esquerdo, sendo que o rim esquerdo apresentou maior porcentagem de fibrose em relação ao rim direito. Os terços do rim esquerdo apresentaram maior porcentagem de fibrose intersticial, com exceção do terço inferior direito, com porcentagem de fibrose de $8,19 \%$. Mas, comparandose os terços do rim direito e esquerdo, observou-se que o terço inferior foi o que apresentou maior porcentagem de fibrose intersticial, respectivamente, 8,19\% e 8,11\% (Tabela 3).

Tabela 4 -
A porcentagem de fibrose do rim direito na faixa etária de 20 a 49 anos variou entre 6,6 e 7,0\% e foi menor quando comparada com a faixa etária acima de 50 anos, em que a média de fibrose intersticial variou entre 8,3 e 8,9\%, predominando um aumento na porcentagem de fibrose com o envelhecimento do indivíduo. Distribuição semelhante foi observada também no rim esquerdo em que a faixa etária acima de 50 anos também apresentou média de fibrose acima de 8,3\% (Tabela 4). A faixa etária de pacientes idosos apresentou maior porcentagem de fibrose tanto no rim direito quanto no rim esquerdo, quando comparados aos pacientes adultos jovens (20 a 39 anos) e adultos (40 a 59 anos), sendo que, no rim esquerdo, a porcentagem de fibrose foi de 9,0\%, maior que a do rim direito (Tabela 5).

A porcentagem de fibrose aumentou com o envelhecimento, mostrando variação de 6,0 a 10,7\% nos terços renais de ambos os rins. A faixa etária de 20 a 39 anos apresentou média de porcentagem de fibrose entre 6,8 e $8,2 \%$, nos terços do rim direito, e entre 7,1 e 9,0\%, nos terços do rim esquerdo. Já a faixa etária de 40 a 79 anos mostrou porcentagem

\begin{tabular}{|c|c|c|c|c|}
\hline Idade (décadas) & $\mathbf{n}$ & $\%$ & Rim direito & Rim esquerdo \\
\hline & & & \multicolumn{2}{|c|}{$X \pm D P$} \\
\hline $20-29$ & 11 & 28,9 & $7,09 \pm 3,01$ & $7,71 \pm 2,93$ \\
\hline $30-39$ & 9 & 23,7 & $8,35 \pm 2,85$ & $8,99 \pm 3,48$ \\
\hline $40-49$ & 6 & 15,8 & $6,60 \pm 4,20$ & $6,58 \pm 2,95$ \\
\hline $50-59$ & 4 & 10,5 & $8,33 \pm 2,35$ & $8,31 \pm 2,17$ \\
\hline $60-69$ & 6 & 15,8 & $8,98 \pm 1,70$ & $9,28 \pm 3,55$ \\
\hline $70-79$ & 2 & 5,3 & $8,97 \pm 1,45$ & $8,23 \pm 0,42$ \\
\hline Total & 38 & 100 & & \\
\hline
\end{tabular}

Rim direito: $F=0,667 ; p=0,651 /$ Rim esquerdo: $F=0,653 ; p=0,661$ $\mathrm{n}=\mathrm{n}^{\mathrm{o}}$ de pacientes

Tabela 3 - Porcentagem de fibrose no compartimento intersticial renal nos pólos do rim direito e esquerdo de 38 pacientes autopsiados no HE-UFTM, Uberaba (MG), no período de janeiro de 2000 a julho de 2004.

\begin{tabular}{crc}
\hline Terços & Rim direito & Rim esquerdo \\
\hline & $\boldsymbol{X} \pm \boldsymbol{D P}$ & \\
Superior & $7,863 \pm 3,373$ & $8,716 \pm 3,555$ \\
Médio & $7,470 \pm 3,221$ & $7,706 \pm 3,887$ \\
Inferior & $8,196 \pm 3,800$ & $8,112 \pm 3,116$ \\
\hline
\end{tabular}

Rim direito: $F=0,416 ; p=0,661 /$ Rim esquerdo: $F=0,785 ; p=0,459$.

de fibrose de 6,0 a 10,4\%, nos terços do rim direito, e de 5,7 a 10,7\%, nos terços do rim esquerdo (Tabelas 6 e 7). Em relação ao rim direito, o terço inferior apresentou maior porcentagem de fibrose ao longo das décadas, se comparado aos terços superior e médio. Essa distribuição não foi semelhante no rim esquerdo, 
em que o terço superior foi o que apresentou maior porcentagem de fibrose ao longo das décadas (Tabelas 6 e 7 ).

Tabela 5 - Comparação entre idade em faixas etárias e fibrose intersticial renal do rim direito e esquerdo de 38 pacientes autopsiados no HE-UFTM, Uberaba (MG), no período de janeiro de 2000 a julho de 2004.

\begin{tabular}{ccccc}
\hline Faixa etária & n & \% & Rim direito & Rim esquerdo \\
\hline & & & \multicolumn{2}{c}{$\boldsymbol{X} \pm \boldsymbol{D P}$} \\
Adulto jovem & 20 & 52,6 & $7,66 \pm 2,93$ & $8,29 \pm 3,17$ \\
Adulto & 10 & 26,4 & $7,29 \pm 3,53$ & $7,27 \pm 2,68$ \\
Idoso & 8 & 21 & $8,98 \pm 1,54$ & $9,01 \pm 3,04$ \\
\hline Total & 38 & 100 & &
\end{tabular}

Rim direito: $\mathrm{F}=0,838 ; \mathrm{p}=0,441$ / Rim esquerdo: $\mathrm{F}=0,767 ; \mathrm{p}=0,472$ $\mathrm{n}=\mathrm{n}^{\mathrm{o}}$ de pacientes

Tabela 6 - Comparação entre idade em décadas e fibrose intersticial renal nos terços superior, médio e inferior do rim direito de 38 pacientes autopsiados no HE-UFTM, Uberaba (MG), no período de janeiro de 2000 a julho de 2004.

\begin{tabular}{ccccc}
\hline Décadas & Terço Superior & $\begin{array}{c}\text { Rim direito } \\
\text { Terço Médio } \\
\boldsymbol{X} \pm \boldsymbol{D P}\end{array}$ & Terço Inferior & \\
\hline $20-29$ & $6,86 \pm 4,03$ & $6,94 \pm 2,96$ & $7,47 \pm 4,32$ & $11(28,9)$ \\
$30-39$ & $8,49 \pm 3,47$ & $8,26 \pm 3,63$ & $8,28 \pm 3,14$ & $9(23,7)$ \\
$40-49$ & $6,75 \pm 3,63$ & $6,05 \pm 4,37$ & $7,02 \pm 5,65$ & $6(15,8)$ \\
$50-59$ & $7,34 \pm 2,96$ & $9,94 \pm 2,70$ & $7,70 \pm 2,28$ & $4(10,5)$ \\
$60-69$ & $9,34 \pm 1,75$ & $7,44 \pm 2,10$ & $10,16 \pm 2,96$ & $6(15,8)$ \\
$70-79$ & $10,42 \pm 2,08$ & $6,17 \pm 1,34$ & $10,32 \pm 0,94$ & $2(5,3)$ \\
\hline Total & & & & $38(100)$ \\
\hline
\end{tabular}

Rim direito (SD: $F=0,048 ; p=0,526 / \mathrm{MD}: \mathrm{F}=0,931 ; \mathrm{p}=0,474$ / ID: $F=0,624 ; \mathrm{p}=0,682$ )

Tabela 7 - Comparação entre idade em décadas e fibrose intersticial renal nos terços superior, médio e inferior do rim esquerdo de 38 pacientes autopsiados no HE-UFTM, Uberaba (MG), no período de janeiro de 2000 a julho de 2004.

\begin{tabular}{ccccc}
\hline Décadas & Terço Superior & $\begin{array}{c}\text { Rim esquerdo } \\
\text { Terço Médio } \\
\boldsymbol{X} \pm \boldsymbol{D P}\end{array}$ & Terço Inferior & $\mathbf{n ( \% )}$ \\
\hline $20-29$ & $8,70 \pm 4,13$ & $7,32 \pm 2,88$ & $7,11 \pm 3,09$ & $11(28,9)$ \\
$30-39$ & $9,03 \pm 4,06$ & $8,98 \pm 2,90$ & $8,98 \pm 4,36$ & $9(23,7)$ \\
$40-49$ & $6,91 \pm 2,75$ & $5,79 \pm 5,75$ & $7,05 \pm 1,53$ & $6(15,8)$ \\
$50-59$ & $7,15 \pm 2,34$ & $9,61 \pm 4,18$ & $8,16 \pm 1,23$ & $4(10,5)$ \\
$60-69$ & $10,76 \pm 3,11$ & $7,64 \pm 5,14$ & $9,43 \pm 3,41$ & $6(15,8)$ \\
$70-79$ & $9,77 \pm 1,94$ & $6,12 \pm 0,15$ & $8,79 \pm 0,52$ & $2(5,3)$ \\
\hline Total & & & & $38(100)$ \\
\hline
\end{tabular}

Rim esquerdo (SE: F= 0,899; $p=0,494 / \mathrm{ME}: \mathrm{F}=0,741 ; \mathrm{p}=0,599$ / IE: $\mathrm{F}=0,712 ; \mathrm{p}=$ $0,619)$ 


\section{Discussão}

Existem razões práticas e teóricas que justificam a importância de se definir parâmetros renais normais em relação à idade, ao gênero, à cor e à estrutura corporal, pois, sem os fatores que determinam as condições normais dos rins não seria possível mensurar estes parâmetros em pacientes portadores de nefropatias (KASISKE; UMEN, 1986).

Houve predomínio do gênero masculino em $63 \%$ dos casos, entre as faixas etárias de 20 a 79 anos. O predomínio do gênero masculino está de acordo com os resultados encontrados por outros autores (LAURENTI; JORGE; GOTLIEB, 2005), em que a proporção de óbitos no gênero masculino na faixa etária de 20 a 79 anos chegou a 73,6\%, no ano de 2001. Neste trabalho, a redução da mortalidade masculina e considerada mais lenta e menor do que a observada no gênero feminino, devido aos padrões diferenciados de morbi-mortalidade que atingem homens e mulheres, já que existem fatores que influenciam muito mais a saúde masculina.

Quanto à cor, houve predomínio dos indivíduos da cor branca, que representaram 58\% dos casos. São ainda pouco mesuradas as informações relacionadas com o impacto da cor, bem como da idade e do gênero sobre a arquitetura renal de indivíduos normais. Estudos mostraram que os indivíduos brancos aparecem em maior número, embora a cor não seja um fator importante na alteração da estrutura renal (KASISKE; UMEN, 1986; MOAR; REINACH, 1988).

Em nosso estudo, a média de idade dos indivíduos foi de 42,7 anos e não mostrou diferença entre os gêneros. Em 1950, a idade média da população na América Latina e Caribe era de 23,5 anos e, em 1999, a média foi de 26,4 anos (CORBINOS, 1950). Segundo esses mesmos autores, a estimativa da média de idade para o ano de 2050 é que será um valor próximo ao encontrado por nosso estudo, correspondente a 37,8 anos. Outros estudos mostraram que a média de idade no gênero masculino é de 56,2 anos e no gênero feminino, de 61,3 anos (NEUGARTEN, KASISKE, SILBIGER et al., 2002).

O aumento considerável do número de idosos na população é observado tanto nos países desenvolvidos quanto nos países em desenvolvimento, como é o caso do Brasil. Nos Estados Unidos, a população de homens brancos de 85 anos e mais representava $0,32 \%$ e $0,74 \%$, respectivamente em 1950 e 1990, devendo-se lembrar que eram inferiores aos valores femininos. No Brasil, entre os homens, as proporções de idosos de 60 anos e mais eram iguais a $5,8 \%, 6,8 \%, 7,3 \%$ e $7,8 \%$, respectivamente, em 1980, 1991, 1996 e 2001, e as de 80 anos e mais eram, respectivamente $0,4 \%, 0,6 \%, 0,8 \%$ e $0,9 \%$, considerando-se os mesmos anos (LAURENTI, JORGE; GOTLIEB, 2005).

A problemática decorrente do envelhecimento, no que diz respeito à saúde, tende a ser a mesma que se verifica nos países desenvolvidos com as doenças crônico-degenerativas, atingindo uma grande parcela da população, agravada, no entanto, pelo fato de persistirem, enquanto prioridades, problemas como desnutrição protéico-calórica e doenças infecciosas (AMARAL, COELI; COSTA et al., 2004).

Em nosso estudo, a média do peso renal total foi de 344g, sendo que, no gênero masculino, a média do peso renal total foi maior do que no gênero feminino. Muitas observações sugerem que os hormônios seriam os responsáveis por determinar as diferenças entre os gêneros, evidenciando que a estrutura renal masculina teria maior peso e comprimento (SAHNI; JIT; SODHI, 2001; NEUGARTEN, KASISKE, SILBIGER et al., 2002). Os valores de peso renais de indivíduos adultos saudáveis evidenciam a variabilidade existente entre os gêneros. O peso renal masculino varia entre 267 e $432 \mathrm{~g}$ e o peso renal feminino varia de 228 a 312g (KASISKE; UMEN, 1986). O peso renal total encontra-se maior no gênero masculino, correspondendo a 368g, do que no gênero feminino, correspondendo a $289 \mathrm{~g}$ (MILETIÉ, FUCKAR, SUSTIÉ et. al., 1998; SAHNI, JIT e SODHI, 2001; NEUGARTEN, KASISKE, SILBIGER et al., 2002).

Já o peso renal total mostrou tendência a diminuir à medida que a idade aumentava, da mesma forma que o peso do rim direito e o peso do rim esquerdo, o que também esteve de acordo com a literatura (MACKAY, 1932; LYE, 1984; KA- 
SISKE; UMEN, 1986; EPSTEIN, 1996; BAYLIS; CORMAN, 1998; RODRIGUEZ-PUYOL, 1998; MILETIÉ, FUCKAR, SUSTIÉ et al., 1998; NEUGARTEN, KASISKE, SILBIGER et al., 2002).

Nos resultados do nosso trabalho, o peso renal esquerdo foi maior que o peso renal direito. Em relação aos gêneros, o gênero masculino apresentou peso renal esquerdo e direito, respectivamente $188 \mathrm{~g}$ e $181 \mathrm{~g}$, maior do que o gênero feminino, o qual não mostrou diferença nos pesos do rim esquerdo e direito. Estudos já realizados confirmam que o peso renal esquerdo supera o peso renal direito, tanto no gênero masculino, respectivamente 111,8g e 108,7g, quanto no gênero feminino, respectivamente 99,4g e 96,6g. Da mesma forma, afirma-se que o gênero masculino possui peso renal maior do que o gênero feminino (MACKAY, 1932; SAHNI; JIT; SODHI, 2001).

Em nosso estudo, a média do IMC foi de $21,4 \pm 5,2 \mathrm{Kg} / \mathrm{m}^{2}$ e não apresentou diferença estatística entre os gêneros, mas houve uma correlação positiva e significativa entre o peso renal total e o IMC, como também entre o peso renal total e o peso corporal $(\mathrm{p}<0,05)$. Outros autores mostraram em estudo que $48 \%$ dos 357 indivíduos analisados apresentaram quadro de obesidade associado ao aumento do peso renal, sendo estatisticamente significante a correlação entre o peso corporal e o peso renal total (KASISKE; UMEN, 1986). Outros autores também confirmaram a correlação positiva entre o peso renal e o peso corporal (MACKAY, 1932; SAHNI; JIT; SODHI, 2001).

A ingesta alimentar exerce influências no desenvolvimento de doenças renais. Em modelos experimentais que foram induzidos a desenvolver doença renal crônica progressiva ainda jovens, a ingesta alimentar normal associada à doença renal não permite ganho de peso adequado para a idade e ainda favorece a progressão de lesões renais. Essas observações levantaram a hipótese de que as lesões renais relacionadas à idade podem estar ligadas à ingesta alimentar, já que o controle da nutrição deve proteger o rim senil de doenças (YU, MASORO, MURATA et al., 1982; MAEDA, GLEISER, MASORO et al., 1985; OWEN; HEYWOOD 1986).

A influência da nutrição na progressão das lesões renais já foi documentada em muitos estudos. O pioneiro, como o realizado por Saxton e Kimball (1942), mostra que a ingestão de dieta com baixas quantidades de proteínas ou a restrição alimentar teriam um efeito protetor contra as lesões renais.

A diminuição da massa muscular esquelética tem sido freqüentemente vista em pacientes com redução da função renal para menos de 30\% do valor normal, o que está relacionado a dietas deficientes em calorias e/ou proteínas, ou ainda a anormalidades metabólicas, ligadas ao alto consumo de dietas protéicas (BALIGA, GEORGE, RAY et al., 1991).

A análise morfométrica da fibrose intersticial nas diferentes idades dos pacientes sem evidências de doenças renais, do nosso trabalho, mostrou uma porcentagem de fibrose intersticial total maior do que 20\% em ambos os rins. Esta também apresentou um aumento em relação à faixa etária, ilustrando, entre os 20 e 79 anos, uma porcentagem de 7 a aproximadamente 9,0\%, no rim direito, e de 7,7 a $9,2 \%$, no rim esquerdo. Em relação à porcentagem de fibrose intersticial nos terços superior, médio e inferior de ambos os rins, observou-se que, em todos os terços, a porcentagem de fibrose intersticial variou entre 6,0 e $10,4 \%$, sendo que o terço inferior do rim direito e o terço superior do rim esquerdo apresentaram maior porcentagem de fibrose em relação aos demais.

De acordo com a literatura, lesões que comprometem a arquitetura renal podem também levar à redução no número de capilares peritubulares, além de induzir aumentada expressão de substâncias vasoconstritoras, como a angiotensina II, levando a um baixo suprimento de oxigênio para o interstício. Existe uma relação entre a perda de capilares peritubulares e o início de processos isquêmicos responsáveis por induzir inflamação e, mais tardiamente, fibrose intersticial (EDDY, 1996).

Observou-se também em nosso estudo que a porcentagem de fibrose intersticial foi maior na faixa etária idosa, correspondendo a mais de 8,5\% em ambos os rins, reforçando que a porcentagem de fibrose tende a aumentar com a idade. Esses dados corroboram com a literatura que afirma que a fibrose no compartimento intersticial é uma altera- 
ção freqüentemente relacionada ao rim envelhecido (DARMADY; OFFER; WOODHOUSE, 1972).

A senilidade vem acompanhada de uma variedade de alterações morfológicas, caracterizada pela atrofia do órgão, diminuição do número de células epiteliais dos túbulos contorcidos, redução do número de néfrons, alterações arteriais, aumento do número de células do interstício renal e fibrose intersticial (TAUCHI; TSUBOI; OKUTOMI, 1971).

Apesar da escassa literatura disponível capaz de associar a estrutura renal com a idade do paciente na ausência de doenças renais, conseguimos concluir, com nossos resultados, que o envelhecimento fisiológico normalmente está associado a alterações macroscópicas e microscópicas relevantes na estrutura renal (KASISKE; UMEN, 1986).

\section{Conclusão}

Apesar das poucas evidências relatadas na literatura sobre alterações estruturais que envolveriam os rins durante o processo do envelhecer do ser humano, nosso estudo mostrou que o peso de ambos os rins tendem a diminuir com o aumentar da idade, tanto no gênero masculino quanto no gênero feminino, apesar de o primeiro ter mostrado média de peso superior.

E mesmo que a hipotrofia renal seja uma alteração morfológica que acompanha o envelhecimento, foi possível analisar que a massa corporal do indivíduo influencia diretamente o peso de ambos os rins, indicando que fatores relacionados à obesidade ou à desnutrição conseguiriam implicar alterações estruturais na fase adulta, que poderiam até desfavorecer a função renal na senilidade.

Sendo assim, o estudo proporciona o entendimento das possíveis alterações do ponto de vista anatômico que os rins podem sofrer no envelhecimento fisiológico, uma vez que alterações de caráter microscópico já são observadas nos quatro compartimentos renais, glomérulos, túbulos, vasos e interstício.

\section{REFERÊNCIAS}

ABRASS, C. K.; ADCOX, M. J.; RAUGI, G. J. Agingassociated changes in renal extracellular matrix. American Journal of Pathology, v.146,n.3, p.742-52, 1995.

AMARAL, A. C. S.; COELI, C. M., et al. Perfil de morbidade e de mortalidade de pacientes idosos hospitalizados. Cadernos de Saúde Pública, v.20, n.6, p.1617-1626, nov-dez, 2004.

BALIGA, R.; GEORGE, V. T., et. al. Effects of reduced renal function and dietary protein on muscle protein synthesis. Kidney International, v.39, p. 831-835, 1991.

BAYLIS, C.; CORMAN, B. The aging kidney: insights from experimental studies. Journal American Society Nephrology, v.2, p. 659-709, 1998.

BECK, L. H. The aging kidney: defending a delicate balance of fluid and electrolytes. Geriatrics, v.55, n.4, p.26-32, 2000.

CORBINOS, L. A. Envejecimiento en América Latina y el Caribe: hechos sociodemográficos y reflexiones éticas. Acta Bioética, v.7, n.1, p. 27-42, 2001.

DARMADI, E. M.; OFFER, J.; WOODHOUSE, M. A. The parameters of the ageing kidney. Journal Pathology, n.109, p. 195-206, 1973.

DUNNILL, M. S.; HALLEY, W. Some observations on the quantitative anatomy of the kidney. Journal of Pathology, n.110, p. 113-121, 1973.

EDDY, A A. Molecular insights into renal interstitial fibrosis. Journal American Society Nephrology. n.7, p. 2495-508, 1996.

EIKMANS, M.; BAELDE, H. J.; HEER, E. et al. Effect of age and biopsy site on extracellular matrix mRNA and protein levels in human kidney biopsies. Kidney International, n.60, p. 974-981, 2001.

EPSTEIN, M. Aging and the kidney. Journal American Society Nephrology, v. 7, p.1106-1122, 1996.

JASSAL, S. V.; OREOPOULOS, D. G. The aging kidney. Geriatric Nephrology and Urology, v.8, p. 141-147, 1998.

KASISKE, B. L.; UMEN, A. J. The influence of age, sex, race and body habitus on kidney weight in humans. Archives Pathology Laboratory Medicine, v.110, p. 55-60, 1986.

LAURENTI, R; JORGE, M. H. P. M., et al. Perfil epidemiológico da morbi-mortalidade masculina. Ciência e Saúde Coletiva, v.10, n.1, p. 35-46, 2005.

LEMLEY, K. V; KRIZ, W. Anatomy of the renal interstitium. Kidney International. n.39, p. 370-381, 1991.

LYE, M. Electrolyte disorders in the elderly. Clinics Endocrinology Metabolism, v.13, n.2, p. 377-398, 1984.

MACKAY, E. M. Kidney weight, body size and renal function. Archives International Medicine, v.50, p.590-594, 1932. 
MAEDA, H.; GLEISER, C., et. al. Nutritional influences on aging of Fisher 344 rats. II Pathology. Journal of Gerontology, v.40, p. 671-688, 1985.

MILETIÉ, D., FUCKAR, Z., et al. Sonographic measurement of absolute and relative renal length in adults. Journal Clinical Ultrasound, v.26, p. 185-189, 1998.

MOAR, J.J.; REINACH, S.G. Renal weights in the southern african black population. American Journal of Physical Anthropology, v.76, p.105-110, 1988.

NEUGARTEN, J., KASISKE, B., et al. Effects of sex on renal structure. Nephron, v.90, p.139-144, 2002.

OWEN, R.; HEYWOOD, R. Age-related variation in renal structure and function in Sprague-dawley. Toxicology Pathology, v.14, p. 158-167, 1986.

RODRIGUEZ-PYOL, D. The aging kidney. Kidney International; v.54, p. 2247-2265, 1998.

SAHNI, D., JIT, I., et al. Weigth and measurements of kidney in Northwest Indian adults. American Journal Human Biology, v.13, p. 726-732, 2001.

TAUCHI, H.; TSUBOI, K.; OKUTOMI, J. Age changes in the human kidney of the different races. Gerontologia, n.17, p. 87-97, 1971.

YU, B., MASORO, E., et al. Lifespan study of SPF Fischer 344 male rats fed ad libitium or restricted diets: longevity, growyh, lean body mass and disease. Journal of Gerontology, v.37, p. 130-141, 1982. 